\title{
New Minimally Invasive Bridge-Plate Technique for Treatment of Atrophic Humeral Shaft Nonunion
}

\author{
Paulo Roberto Vilaca Junior, ${ }^{1, *}$ Americo Zoppi Filho, ${ }^{1}$ and Farshid Moshiri ${ }^{2}$ \\ ${ }^{1}$ Shoulder and Elbow Group, Beneficent Association Nossa Senhora do Pari/ Pari Hospital, Sao Paulo, Brazil \\ ${ }^{2}$ Necker Hospital, Paris, France \\ "Corresponding author: Paulo Roberto Vilaca Junior, Shoulder and Elbow Group, Beneficent Association Nossa Senhora do Pari/ Pari Hospital, Sao Paulo, Brazil. Tel. \\ +55-1133226500, E-mail: prvjbr@gmail.com
}

Received 2015 August 30; Revised 2015 September 23; Accepted 2015 October 03.

\begin{abstract}
Introduction: Humeral nonunion is a condition resulting from lack of healing at the fracture site often associated with alteration of the local biological potential.

Case Presentation: A 38-year-old male patient, who had a fracture in the left humerus caused by a fall 8 months earlier was presented. He was treated non-operatively with Sarmiento brace.

Conclusions: The present study introduced a new minimally invasive bridge-plate technique for treatment of humeral shaft nonunion that avoids wide dissection, radial nerve isolation and periosteum stripping. It is a safe procedure, which led to bone healing and good results in a patient with atrophic humeral shaft nonunion.
\end{abstract}

Keywords: Humerus, Nonunion, Minimally-Invasive Surgery

\section{Introduction}

Humeral nonunion is a condition resulting from lack of healing at the fracture site often associated with alteration of the local biological potential. Treatment is a challenge due to preoperative conditions and difficulties inherent to the surgery.

Ring et al. (1) demonstrated that the most important factor to achieve bone healing in nonunion is use of a careful, biologically and mechanically adequate technique.

Using a surgical technique that respects the basic principles of less soft tissue dissection, preservation of blood supply and immediate rehabilitation of the operated limb is respected. The present study introduced an original surgical technique for treatment of humeral shaft nonunion and the results in a patient with atrophic humeral shaft nonunion.

\section{Case Presentation}

\subsection{Operative Technique}

A 38-year-old male patient, right-handed, with athrophic nonunion (Figure 1) who had a fracture in the left humerus caused by a fall 8 months earlier was referred. He was treated non-operatively with Sarmiento brace. At this visit, he was operated in supine position following previously described technique (2-4), with the bone graft removed from the contralateral iliac crest in case of atrophic nonunion. In distal humerus region, a four-cm anterior longitudinal incision was made on the lateral border of the biceps. Between the biceps and the brachioradialis muscles, the brachial muscle could be accessed more deeply. A blunt longitudinal midline opening was made in the fibers of the brachial muscle.

The brachialis muscle is innervated at the lateral surface by the radial nerve and medially by the musculocutaneous nerve. This anatomical detail allows its longitudinal opening without any harm to nerves or loss of function; then, access to the anterior surface of the humerus is easily achieved. Through this opening, the screws would be fixed distally.

Distally, the muscles including the lateral part of brachialis and brachioradialis protect the radial nerve, which is neither seen nor dissected in this approach. Proximally, a four-cm incision is made between the medial border of deltoid and lateral border of biceps.

The interval between these two muscles is the location to slide in and fix the plate. A 4.5-mm narrow dynamic compression plate (DCP) must be slid on the anterior surface of the humerus, submuscular to the brachial muscle. The direction in which the plate is slid differs according to each case. Distally, one must be careful not to violate the coronoid fossa.

With the plate slid over the anterior surface of the 
humerus, the screws (two in each fragment) are inserted from anterior to posterior on the ends of the plate.

An one-cm auxiliary incision is made over the nonunion site on the anterior surface of the humerus with direct dissection to the site (Figure 2). An arthroscopic canula is inserted in this incision and kept in contact with the nonunion site. A Kirchner wire is passed through the canula and located in the nonunion site. A scope image was made to confirm the correct position of the canula. Canula must be left in that position until the end of the procedure. After the removal of the Kirchner wire, a shaver blur is introduced and with it, fibrous tissue is removed (Figure 3). A cancellous iliac autologous graft is inserted through the canula into the nonunion site (Figure 4).

\subsection{Rehabilitation}

Immediately postoperatively, patient was directed to use the limb in his usual activities and use a sling only for pain control in the first five days if necessary, mainly at night when sleeping. Use of immobilization was not advised after this period.

He was recommended to move the elbow and shoulder, avoiding stiffness. After bone healing, a rehabilitation program was performed. The first aim was to gain full mobility, then proprioception and finally muscular strengthening. The total rehabilitation period depends on the progression of patient. The final goal was to restore full range of motion and strength without pain. Bone healing was obtained after three months postoperatively (Figure 5). There were no nerve injuries or infection.

\section{Discussion}

This case shows that functional results after humeral shaft nonunion can be obtained with a minimally invasive approach. This technique has certain advantages that make it appealing to the trauma surgeon.

Plates can be safely used anteriorly along the humerus with this technique. Good results have been achieved with submuscular plating with no major soft tissue problems and with functional results similar to other methods in the literature. Previous studies demonstrated faster recovery with minimally invasive techniques compared with invasive open technique (5-8).

The healing of the humeral shaft fracture in this case presents good results with the advantage of using a minimally invasive technique. This fixation aims at maintaining bone alignment through indirect reduction without an open approach to the fracture site. This preserves local blood supply and results in less surgical damage to soft tissues, replacing absolute stability by relative stability to achieve bone healing by stimulating bone formation.

There are several studies in the literature showing the advantages of using a bridge-plate in fractures of different parts of the body, such as femur and tibia, but there are few reports of use of this technique for humerus $(4,6)$.

Moreover, there is lack of reports in the literature on use of this technique for nonunions $(2,3)$. Being a minimally invasive technique, complications are reduced (5, $7,8)$. There were no nerve injuries. The bridge-plate technique for treatment of humeral shaft nonunion is indicated for both atrophic and hypertrophic nonunions.

The objective of the present case report was not to compare techniques or healing times between atrophic or hypertrophic nonunions, but to demonstrate the possiblity to use a minimally invasive technique for nonunion and present the results as an alternative to the traditional technique.

It was shown here that there is a biological capacity for humeral shaft healing achieved through the bridge-plate technique, with use of bone grafting. It is not necessary to have absolute stability or focal compression, contradicting previous works (7).

Without the need for broad dissection, the local blood supply is preserved. By combining biological stimulus promoted by bone graft and sufficient mechanical stability granted by the plate, all elements necessary for healing of nonunion are present (7). The present technique makes a difficult procedure much easier by avoiding wide dissection, radial nerve isolation and periosteum stripping.

The results obtained confirm the conclusions of Ring et al. (1), who stated that it is important to use a careful, biologically and mechanically adequate technique to achieve bone healing. With the plate slid over the anterior surface of the humerus and respecting the approaches previously described, the radial nerve is totally protected $(2-4,6)$ and there is no inherent danger to any vascular structure.

It is important, in future studies, to identify the limitations of the technique and define whether it is applicable for more severe cases, as well as possible alternatives to the use of autologous bone graft.

In conclusion, this case report demonstrated that this new anterior minimally invasive bridge-plate technique for treatment of humeral shaft nonunion presents satisfactory results regarding bone healing and functional capacity. 
Figure 1. Humerus in Lateral View

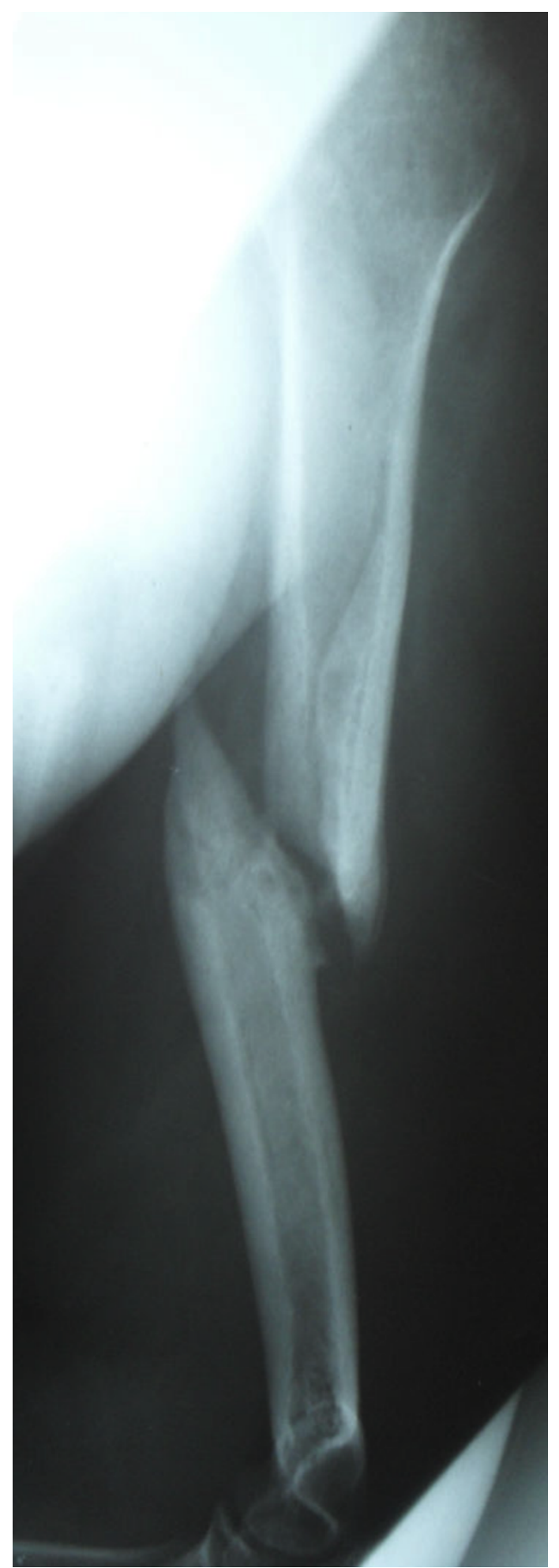

Atrophic nonunion.

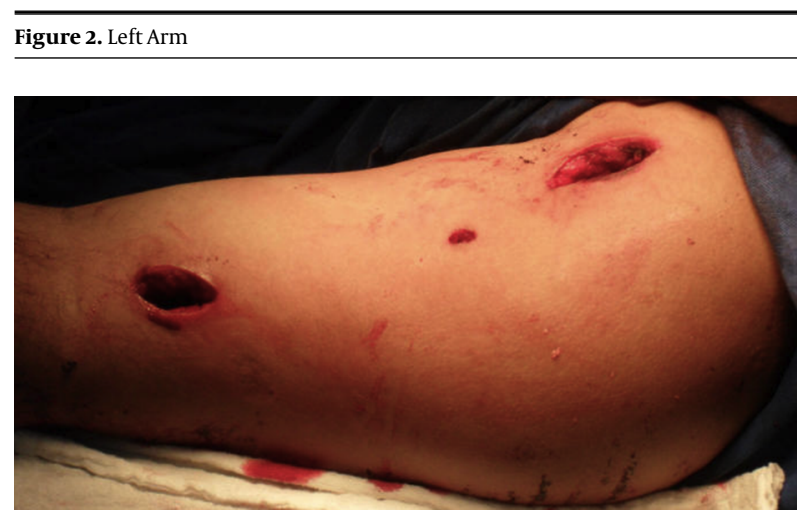

Proximal and distal incisions for plate introduction and screw fixation; an auxiliary incision on the anterior surface of the humerus for the canula introduction.

Figure 3. Intraoperative Scope Image

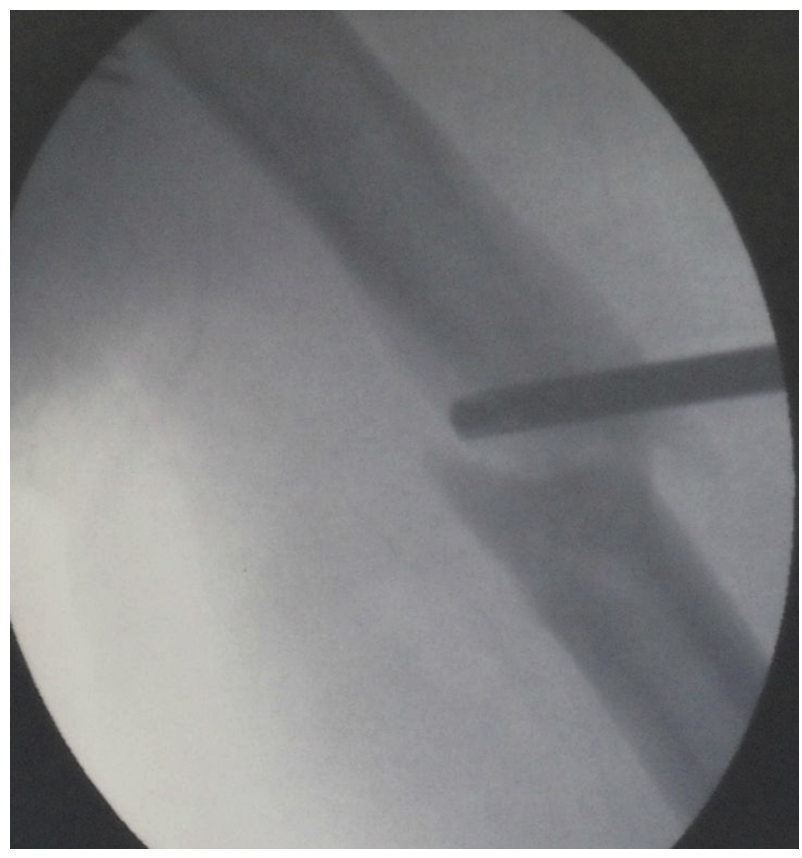

Fibrous tissue is removed with a shaver blur 


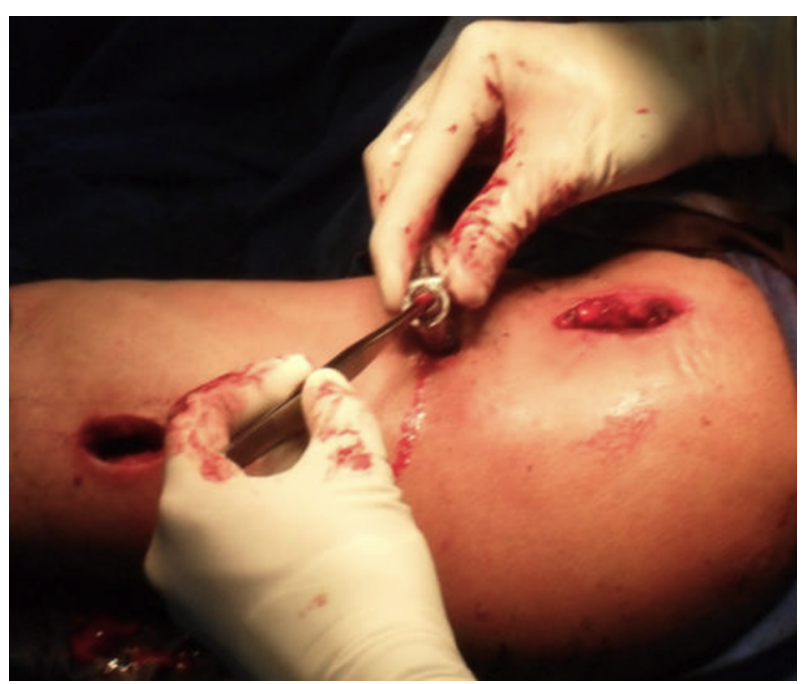

Figure 4. The Bone Graft is Inserted into the Atrophic Nonunion Site Through the Canula.
Figure 5. Humerus in Lateral View

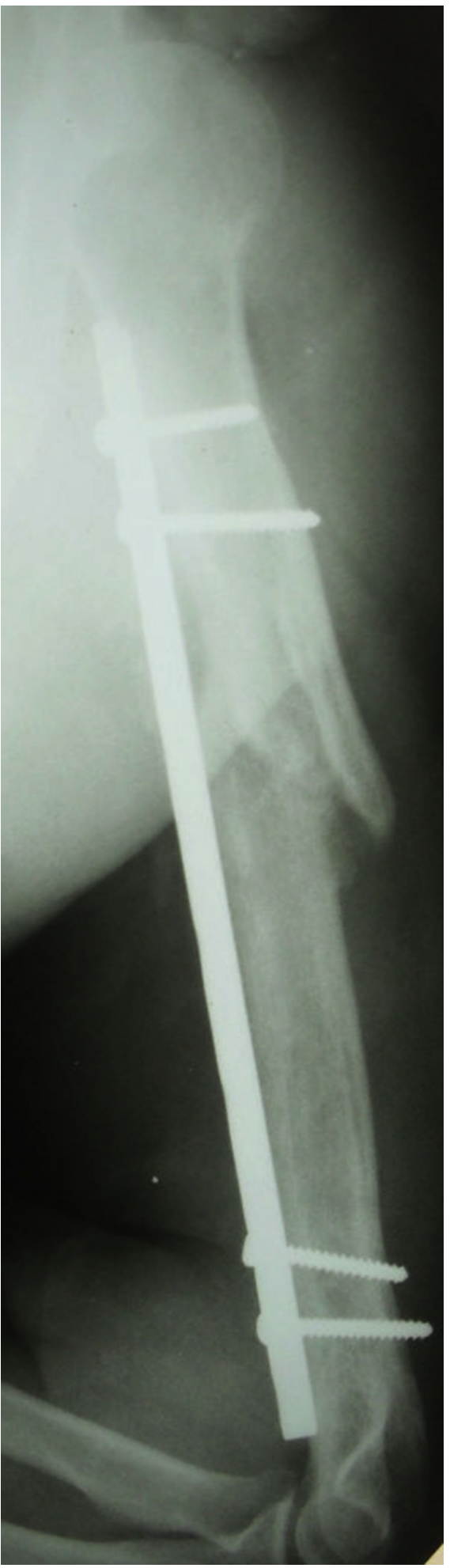

Bone consolidated. 


\section{References}

1. Ring D, Jupiter JB, Quintero J, Sanders RA, Marti RK. Atrophic ununited diaphyseal fractures of the humerus with a bony defect: treatment by wave-plate osteosynthesis. J Bone Joint Surg Br. 2000;82(6):867-71. [PubMed: 10990313]

2. Vilaca PJ, Uezumi MK. Anterior minimally invasive bridge-plate technique for treatment of humeral shaft nonunion. J Orthop Traumatol. 2012;13(4):211-6. doi: 10.1007/s10195-012-0203-1. [PubMed: 22717617].

3. Vilaca Junior JR, Uezumi MK. Tratamiento mínimamente invasivo de las pseudoartrosis de la diafisis del humero [In Spanish]. Trauma. 2011;22(3):168-73.

4. Livani B, Belangero WD. Bridging plate osteosynthesis of humeral shaft fractures. Injury. 2004;35(6):587-95. doi: 10.1016/j.injury.2003.12.003. [PubMed: 15135278].

5. Marti RK, Verheyen CC, Besselaar PP. Humeral shaft nonunion: evaluation of uniform surgical repair in fifty-one patients. J Orthop Trauma. 2002;16(2):108-15. [PubMed: 11818806].

6. Zhiquan A, Bingfang Z, Yeming W, Chi Z, Peiyan H. Minimally invasive plating osteosynthesis (MIPO) of middle and distal third humeral shaft fractures. J Orthop Trauma. 2007;21(9):628-33. doi: 10.1097/BOT.0b013e31815928c2. [PubMed: 17921838].

7. Segonds J, Alnot J, Masmejean E. Iconography: Pseudarthroses et retards de consolidation aseptiques de la diaphyse humerale [in French]. Rev Chir Ortho. 2003;89:107-14.

8. King AR, Moran SL, Steinmann SP. Humeral nonunion. Hand Clin. 2007;23(4):449-56. doi: 10.1016/j.hcl.2007.09.003. [PubMed: 18054672] vi. 